\title{
Translational genomics and precision medicine: Moving from the lab to the clinic
}

DOI:

10.1126/science.aax4588

\section{Document Version}

Accepted author manuscript

Link to publication record in Manchester Research Explorer

\section{Citation for published version (APA):}

Zeggini, E., Gloyn, A. L., Barton, A. C., \& Wain, L. V. (2019). Translational genomics and precision medicine: Moving from the lab to the clinic. Science, 365(6460), 1409-1413. https://doi.org/10.1126/science.aax4588

\section{Published in:}

Science

\section{Citing this paper}

Please note that where the full-text provided on Manchester Research Explorer is the Author Accepted Manuscript or Proof version this may differ from the final Published version. If citing, it is advised that you check and use the publisher's definitive version.

\section{General rights}

Copyright and moral rights for the publications made accessible in the Research Explorer are retained by the authors and/or other copyright owners and it is a condition of accessing publications that users recognise and abide by the legal requirements associated with these rights.

\section{Takedown policy}

If you believe that this document breaches copyright please refer to the University of Manchester's Takedown Procedures [http://man.ac.uk/04Y6Bo] or contact uml.scholarlycommunications@manchester.ac.uk providing relevant details, so we can investigate your claim.

\section{OPEN ACCESS}




\title{
Translational Genomics and Precision Medicine: moving from the lab to clinic
}

\author{
Eleftheria Zeggini ${ }^{1}$, Anna L Gloyn ${ }^{2,3,4}$, Anne C Barton ${ }^{5,6}$, Louise V Wain ${ }^{7,8}$ \\ ${ }^{1}$ Institute of Translational Genomics, Helmholtz Zentrum München - German Research Center for \\ Environmental Health, Neuherberg, Germany \\ ${ }^{2}$ Oxford Centre for Diabetes Endocrinology \& Metabolism, Oxford University, Oxford, UK \\ ${ }^{3}$ Wellcome Centre for Human Genetics, University of Oxford, Oxford, UK \\ ${ }^{4}$ Oxford NIHR Biomedical Research Centre, Churchill Hospital, Oxford, UK \\ ${ }^{5}$ Versus Arthritis Centre for Genetics and Genomics, Centre for Musculoskeletal Research, \\ Manchester Academic Health Science Centre, University of Manchester, Manchester, UK \\ ${ }^{6}$ NIHR Manchester Biomedical Research Centre, Manchester University NHS Foundation Trust, \\ Manchester, UK \\ ${ }^{7}$ Department of Health Sciences, University of Leicester, Leicester, UK \\ ${ }^{8}$ National Institute for Health Research, Leicester Respiratory Biomedical Research Centre, Glenfield \\ Hospital, Leicester, UK
}

\section{ABSTRACT}

Translational genomics aims to improve human health by building on discoveries made through genetics research and applying them in the clinical setting. This progress has been made possible by technological advances in genomics, analytics and the digital revolution. Such advances should enable the development of prognostic markers, tailored interventions and the design of prophylactic preventative approaches. We are at the cusp of predicting disease risk for some disorders by means of polygenic risk scores integrated with classical epidemiological risk factors. This should lead to better risk stratification and clinical decision-making. A deeper understanding of the link between genome-wide sequence and association with well-characterised phenotypes will empower the development of biomarkers to aid diagnosis, inform disease progression trajectories and to allow better targeting of treatments to those patients most likely to respond.

\section{INTRODUCTION}

Progress in the field of human genetics has been accelerated by recent technological advances, which allow the genome-wide interrogation of individual and population-wide sequence variation. This has led to the identification of new variants for Mendelian disorders already impacting clinical care; for more common complex diseases, where multiple genetic and environmental effects combine to increase disease risk, thousands of genetic susceptibility variants have been identified. Translation of these findings into improvements in healthcare will require moving from association signal discovery to functional interpretation of that signal, and ultimately to clinical treatment, facilitated by large-scale data generation; the development of advanced computational toolkits to process the wealth of information; addressing ethical, legal, social and economic considerations; and effective integration of genomics into routine clinical practice.

The translation of improvements in genetic understanding to healthcare can be seen for some rare monogenic diseases where precision medicine, defined as an approach to allow more accurate prediction of the groups of people who will benefit from a specific treatment or prevention strategy for a particular disease, is already being used clinically. For example, ivacaftor is a drug that acts at the cystic fibrosis transmembrane conductance regulator channel to alter activity, and is licensed for use in the $4-5 \%$ of cystic fibrosis patients with specific gating mutations in the gene encoding the protein (1). Cancer research is using precision medicine to target therapies according to tumour mutations and has led the field in translating insights from genomics into a better understanding of the mechanisms of disease and novel drug development. For example, genome sequencing coupled with multi-omics-based molecular profiling has led to the development of personalised 
interventions, such as CAR-T Immunotherapy (2). For common diseases, which are caused by combinations of multiple genetic and environmental factors, progress has been slower. However, genetically-driven approaches are now starting to emerge; for example, recent studies have identified variants influencing efficacy (3) and tolerance (4) of metformin, a first-line treatment for type 2 diabetes, and have the potential to impact patient care.

In this review, we focus on complex disease genetics and explore how genetic and genomic discoveries can fuel the translational pathway to improve human health [Fig. 1].

\section{HOW CAN GENETIC STUDIES TRANSLATE INTO CLINICAL APPLICATION? Precision medicine}

One of the immediate clinical applications arising from studies of complex disease genetics is the improved targeting of available therapies to those most likely to respond, or avoidance of therapy in those likely to develop adverse events. Information on an individual's genetic makeup can be used to estimate their likelihood of developing disease, their likely benefit if targeted for preventative care, or aid selection of the best treatment for that individual. Genetic studies to inform precision medicine in the context of best treatment include both 'pharmaco-genomic' studies (the identification of genetic variants that influence drug pharmacokinetics) and genetic studies that strive to define disease 'endotypes' that might reflect different underlying disease aetiopathologies that lie under the same diagnostic umbrella but with different optimal treatment strategies.

Most common complex diseases exhibit a variable disease course and response to therapy; of the 10 best-selling drugs in the US, between 4 and 25 patients are treated in order to achieve one patient with a good response (5). This is not only a burden on health resources, but exposes those patients who are treated without achieving a clinically significant response to the risk of harm through development of adverse events or through delays in switching to alternative treatments that might achieve benefit. This illustrates the need to develop better ways to target therapies.

Initiatives in the US (6) and Europe (7) have been established to drive forward the precision medicine agenda, but progress has been relatively slow. One limiting factor is the measurement of progression or treatment response, often based on clinical end-points rather than biomarkers. Genetic studies can identify which components of the outcome measures are heritable and, therefore, predictable, which may help in developing outcomes that are more objectively measured (8). A further challenge in identifying predictors of drug response is poor drug adherence, which is common in chronic complex diseases. As at least 1 in 4 patients do not take medication as prescribed (9), and this degree of misclassification impacts the power of genetic and other biomarker studies to identify predictors of response. Despite these challenges, some genetic markers of drug response are emerging, such as the associations of HLA-C*06:02 with biologic therapy response in psoriasis (10), and HLA-DRB1 with severity, mortality and treatment response to biologic drugs in rheumatoid arthritis (11).

Future precision medicine studies will consider the impact of non-response and non-adherence in the context of both pharmaco-genomic studies and the emerging recognition of disease endotypes across most common complex diseases. The use of biomarkers as outcome measures, rather than clinical endpoints, will provide objective measures of response that more closely reflect the underlying biological processes in each patient.

\section{Functional genomics and novel drug target discovery}

Genome-wide association studies (GWAS) in complex diseases have been successful in identifying robust associations between specific sequence variants and the phenotypes of interest. Individually, these associations characteristically confer modest to small effects, although there are notable 
exceptions, including the HLA alleles, which tend to exert large effects in autoimmune disease susceptibility in particular (12). In addition, rare or population-specific common variants have been associated with large effects on complex traits like osteoarthritis (13) and type 2 diabetes (14). However, even if the genetic effect is small, it can be informative in terms of drug development pipelines; indeed, recent studies suggest that genes with underlying genetic support for an association with disease are twice as likely to encode successful drug targets in clinical development $(15,16)$. Whilst the most widely-described examples of drug targets with genetic support arise from familial rare disease studies, where rare variants with high penetrance and large effects result in disease (for example, PCSK9) (17), GWAS signals increasingly implicate genes which encode known drug targets [Figure 2]. This provides retrospective proof-of-concept of the ability of GWAS to identify potentially new druggable targets. Furthermore, GWAS have identified drug repurposing opportunities, i.e. targets for which there are already approved drugs, for other indications $(18,19)$, which might be effective in treating an alternative disease.

The GWAS catalog currently contains approximately 138,000 genetic association signals with disease status or quantitative phenotypes from 4000 studies. Whilst each of these signals can act as a signpost to important genes and pathways, making the link between the signal and the specific gene(s) underlying the association has remained a major bottleneck. Although some signals very clearly implicate a particular gene, for example, via an experimentally-validated functional amino acid change or introduction of a stop codon, the majority of genetic signals are either very broad, encompassing many variants across multiple genes, or are outside of gene regions entirely, suggesting a regulatory effect for which the target gene can be difficult to identify.

Computational (in silico) approaches can be used to map signals to effector genes; the availability of increasingly large sample sizes, deeply-sequenced reference panels that allow variants not captured by genotype arrays to be imputed, and samples from ethnically diverse populations allow genetic association signals to be refined to smaller regions. However, it can still be challenging to determine which gene is being regulated and which variant is responsible. A genetic variant regulating gene expression may be functional and will likely reside in a region of open chromatin, but this may vary according to cell type and stimulatory condition. The increasing availability of multi-omics data, such as transcriptomics, proteomics, chromatin accessibility and histone modifications, across multiple cell types and tissues, and under different conditions, now facilitates advances in linking genetic signals to genes. International efforts such as the Genotype-Tissue Expression (GTEx) project, ENCODE, ROADMAP and BLUEPRINT (20-22) have provided publically available resources for studying tissue-specific gene expression and regulation, which can be leveraged to identify effector transcripts for GWAS signals (23). Interrogation of gene and protein expression data, annotation of epigenetic markers indicative of regulatory genomic regions, and chromosome interactions have identified potentially causal variants and genes for a large number of GWAS signals [Figure 3]. For example, in type 2 diabetes, functional annotation of genetically associated variants in human pancreatic islet cells enabled the fine-mapping of $20 \%$ of disease loci (24). Whilst these give insight into the underlying mechanisms of the genetic signal and inform the design of targeted molecular experiments, they are often limited by the range and sample size of molecular data and diseaserelevant tissues that are available, as well as by reproducibility [Figure 2]. The challenge now is to extend these efforts to less accessible disease-relevant cell types, at different developmental stages and under different stimulatory and disease-relevant conditions to allow integration with large-scale genetic discovery efforts. The recently established Human Cell Atlas (HCA) is focused on providing greater resolution by generating reference maps of all human cells using both emerging single-cell 'omics and whole intact-tissue methodologies (25). Acceleration of progress in development of approaches such as high-throughput screening and genetic manipulation coupled to cellular phenotyping is needed. 
Access to certain human tissues remains a challenge but the emergence of protocols for in vitro differentiation of human induced pluripotent stem cells, iPS cells, which can be differentiated into otherwise inaccessible cell types, offers the potential for disease-associated variants to be investigated in appropriate cell types. However, these studies are new and we are still learning much about how representative these systems will be for population level medical applications. Together, these efforts will accelerate translation of GWAS findings to therapeutic interventions through increased confidence in target identification.

\section{Prediction, prevention, prognosis}

Common complex disease risk is conferred potentially by up to thousands of variants prompting development of polygenic risk scores to capture the likelihood of developing disease. As these variants are usually common in frequency with individually small contributions to overall risk, combining information from multiple variants, weighted by their effect size, has the potential to improve on the predictive value of clinical data alone. Genetic risk scores (GRS) have been developed that comprise only signals that pass stringent criteria or that capture a greater proportion of genome-wide risk information (often termed polygenic risk scores). The choice of variants to include in the score is determined by the intended use of that score (for example, to demonstrate the combined effect of specific genetic risk factors to overall disease risk or development of a predictive tool for clinical use) and their power is determined by the number of variants included in the score, the frequency of those variants in the population, the choice of weighting and the contribution of environmental risk factors.

A recent study by Khera et al. (26), demonstrated the power of polygenic risk scores in the context of five common disease showing that around $20 \%$ of the population had a polygenic three-fold increased risk of one or more common diseases; comparable to levels of risk conferred by rare disease-causing variants of high penetrance (26). A GRS for chronic obstructive pulmonary disease (COPD) demonstrated an almost five-fold relative risk when comparing high vs low genetic risk score deciles and, when this was combined with absolute risk estimates for COPD amongst smokers, an absolute risk of COPD of $82.4 \%$ for smokers in the highest risk score decile (compared to $17.4 \%$ for those in the lowest decile) (27). GRS for obesity (28) and blood pressure (29), which are common risk factors for multiple diseases such as cardiovascular disease and type 2 diabetes, and considered to be modifiable, have also demonstrated substantial variation across the population. From a public health perspective, the extent to which these risk factors can be modified on a background of high genetic predisposition, and the most effective means of implementing such modifications, are research areas that should be prioritised to accelerate implementation.

The utility of GRS in population-based genetic screening as a preventive strategy for complex diseases depends on the frequency of the disease being investigated as well as the genetic risk conferred by the associated variants. It is likely to be useful for highly prevalent complex conditions such as coronary artery disease, which affects between 10 and $20 \%$ of the population in western countries (30). However, for some complex polygenic diseases with a low background prevalence ( $<5 \%$ population), even those at the highest genetic risk are more likely not to develop disease than to develop it: for example, in a disease with a population prevalence of $1 \%$ (such as rheumatoid arthritis), even those with a 20 -fold increased risk will still have an $80 \%$ chance of not developing the condition based on genetic risk alone. Additionally, as there are more individuals at lower genetic risk in the population, it has been shown that the majority of cases within the population will come from people at lower genetic risk for disease $(31,32)$. Age-related macular degeneration is a case in point: it affects $5 \%$ of the $>75$ year-old population and was one of the first conditions in which GWAS identified genetic associations, with two genetic susceptibility variants conferring a 50 -fold risk of disease development. However, only $20 \%$ of patients carry high-risk genetic variants, meaning that the majority of cases come from those with lower genetic risk and genetic screening is not currently 
included in guidelines $(33,34)$. Nonetheless, genetic information adds value over and above clinical characteristics in predicting progression of macular degeneration, particularly in those with a less severe disease stage (35).

As such, implementation of GRS for targeting of disease screening requires careful consideration in terms of which thresholds of risk are selected as the basis for screening decisions and the added value beyond clinical information alone (36). For common complex diseases such as stroke and coronary heart disease, where both genetic and environmental factors contribute to disease risk, environmental factors can have a greater influence $(37,38)$. Modifiable environmental risk factors mean that identifying those at high genetic risk of developing disease can still have utility in effecting behavioural change. In a randomised control trial in asymptomatic first degree relatives of patients with rheumatoid arthritis, disclosure of genetic risk information led to positive behaviour modifications including increased smoking cessation and better dental hygiene, both of which are environmental risk factors (39). As GRS tests may call for lifestyle adaptations in response to risk of individual diseases, further research is required to evaluate how to implement best practices to facilitate such changes.

Beyond identifying those at highest risk of developing disease, GRS can also inform development of precision medicine approaches for earlier and more effective treatment by identifying those with the disease who are at highest risk of rapid progression or of more severe manifestations of disease $(26,40)$. Furthermore, using disease-relevant quantitative traits to define the underlying biological processes characterising the disease in an individual holds the promise of assisting with patient stratification; for example patients with type 2 diabetes largely due to insulin secretory defects may respond better to therapies focused on restoring insulin secretion as opposed to improving the action of insulin in target tissues (40). Such studies require large prospective collections of patient samples with high-quality phenotype data to define disease trajectories and/or classify disease subtypes, and will be essential if GRS are to inform clinical care.

The implications for potential additional burden on clinical services of any genetic testing also requires consideration if advances are to translate to the clinical setting. For example, introducing genetic testing for variants that affect Warfarin metabolism in routine clinical care of patients receiving Warfarin anticoagulation reported benefits (41). However, the 45 minutes required to obtain results affected the clinic workflow and potentially required a re-organisation of service delivery (41).

GRS have numerous potential applications in prediction and prevention, but care in their design and implementation is warranted, for example with respect to transferability across and within populations (42). Most GRS to date have been developed using data from European-ancestry studies and although there is support for some generalisability of such scores across ancestries, it is evident that predictive power decreases with ancestral divergence and variation in minor allele frequencies as well as differences in relevant environmental exposures $(27,43)$. If GRS are to be incorporated into clinical decision-making or public health interventions, development of ancestry and population-specific GRS are vital to ensure equity in healthcare and optimal benefit to patients.

\section{HOW CAN THE DISCOVERY PIPELINE BE ACCELERATED? Genomics in diverse populations}

The majority of genetic association studies have focused on people of European ancestry. This not only leads to a bias in our understanding of genetic disease but also results in a fundamental gap as different population characteristics can help identify and fine-map causal variants. At one end of the spectrum, isolated (founder) populations demonstrate high levels of genetic similarity (44), while African populations are characterised by high levels of genetic diversity (45). Thus, in isolated 
populations, protective or deleterious variants that are rare in the general population may be increased in frequency and thus be more easily detected. In contrast, the highly diverse African populations can help determine the architecture of complex disease through fine-mapping and reveal associations that have been missed due to differences in allele frequency between populations (45). For example, a G6PD variant that is common (minor allele frequency $11 \%$ ) in African populations but rare or absent in European populations is associated with decreased $\mathrm{HbA} 1 \mathrm{C}$ levels independently of blood glucose levels. As raised HbA1c is a diagnostic marker for type 2 diabetes, a chronic condition that affects over 400 million people globally, this could lead to substantial under-diagnosis of type 2 diabetes in African ancestry populations if based on $\mathrm{HbA1c}$ levels alone (46). Going forward, the next frontier in genomic medicine will require embracing whole genome sequencing across all global populations to capture and understand the full spectrum of genetic variation and disease associations.

\section{Deep phenotypic characterisation}

Retrospective and prospective longitudinal linkage to electronic health records (EHRs) in cohorts with genetic data can lead to better clinical characterisation of the study population and enable discovery of new genetic associations with disease, disease subtypes and disease-relevant quantitative phenotypes. Several large-scale biobanks and population-based cohorts, such as the UK Biobank (500,000 participants), All of US initiative (1,000,000+ participants) and the Million Veterans Programme, now include linkage to EHRs (47). There are already successful translational examples of coupling genomics to EHRs; for example, identification of loss-of-function mutations in ANGPTL3 associated with the development of coronary artery disease, and subsequent development of an inactivating monoclonal antibody offering promise for disease prevention (48).

Unlike information and measurements collected in a research cohort setting, clinically-derived EHRs have not been recorded with research in mind and are, therefore, even more prone to bias, inconsistency and noise. Differences in how healthcare is delivered, for example via a universal system (like the National Health Service [NHS] in the UK) or via multiple independent providers (as in the USA), leads to differences in how the same condition might be recorded across countries, even when international coding systems are used. Particularly for fragmented healthcare systems, completeness of records is highly variable and this limits their utility to address many research questions, including those related to longitudinal measures. Even within a single system such as the NHS, regional differences in terms of coding exist and can vary from practice to practice and hospital to hospital, and detailed disease-specific records, such as diagnostic scans, are not yet fully captured by routine linkage in many healthcare systems. Furthermore, an understanding of the mechanisms that drive coding decisions is needed to identify potential sources of bias (for example, the Quality and Outcomes Framework in the NHS whereby reward and incentivisation of General Practitioners influences coding practice). These variations exist not only across regions but also over time as coding systems, such as the World Health Organisation International Classification of Disease, are updated.

Deeper phenotyping can also be achieved in research cohort settings through recall of participants. For example, imaging is being undertaken in 100,000 participants in UK Biobank and imaging-derived traits can serve as markers of biological processes with a genetic underpinning, and boost discovery in genetic association studies (49). Many cohorts now have consent to recall participants on the basis of either their phenotype (i.e. disease status) or genotype (for example, carriers of a putative high risk variant), thereby providing further opportunities to undertake deeper or more specific phenotypic characterisation to address specific hypotheses. Recall-by-genotype studies are an exciting new tool when investigating causality in disease (50). The establishment of large-scale biobanks and registries geared towards genomics (as elegantly exemplified by the Nordic countries (51)) also means that previously difficult-to-reach genetic effects, such as gene-environment and gene-gene interactions (e.g. (52)) can be further explored. 
The ability to refine phenotypes has clear benefits in our understanding of disease and disease endotypes but the temptation to divide participants into smaller and smaller subgroups also runs the risk of reducing sample size and consequently, statistical power, for genetic discovery and so a balance must be struck. Furthermore, as phenotype definition becomes more nuanced and narrow, the potential for the variation in coding practices in EHR described above to impact on interpretation becomes more pronounced. Pragmatic definitions that capture the phenotype being studied with reasonable precision and minimal misclassification yet at the same time maximise sample size might be the optimal strategy for genetic association discovery where statistical power is a major consideration, in part due to the large multiple testing burden of genome-wide analyses. Stricter phenotyping, such as that appropriate for descriptive classical epidemiological studies and clinical practice, could then be applied post hoc to delve deeper into the associations that are identified.

In summary, genetic study designs that will be optimal to reap the rewards of deeper phenotyping will ideally require multidisciplinary collaboration that includes both genetic and classical epidemiology expertise as well as clinical insight. Researchers must be cognizant of subjective influences on phenotype definitions in large-scale EHR resources and take an integrative approach that also combines measures that might be less prone to bias (or at least, prone to different biases), for example, biomarkers. The use of carefully considered sensitivity analyses to test the impact of assumptions made and misclassification, will continue to be best practice in these studies.

\section{CONCLUSION}

A better understanding of the genetic aetiology of complex diseases can provide novel insights into fundamental biology and translational opportunities. In recognition of this translational potential, there is a rising number of high-profile, large-investment initiatives focused on genomics in medicine. These traverse public and private funding mechanisms to generate the large-scale clinical and biodata resources needed to spur innovation for personalised medicine and population health. This new, eagerly-awaited digital health era is now becoming a tangible prospect. To ensure that the potential is realised, we recommend focussing on biological outcome measures where possible; accounting for non-adherence in studies of treatment response or ascertainment bias in prognostic studies; collection of prospectively recruited cohorts across diverse ancestries and with linkage to electronic health records so that population-specific PRS can be developed for a range of diseases; finally, investment into how to implement genetic testing as a diagnostic test or to effect behaviour change is essential.

\section{ACKNOWLEDGEMENTS}

Funding: ALG is a Wellcome Trust Senior Fellow Basic Biomedical Science. ALG is funded by the Wellcome Trust (095101, 200837, 106130, 203141), Medical Research Council (MR/L020149/1), European Union Horizon 2020 Programme (T2D Systems), and NIH (U01-DK105535; U01-DK085545) and by the National Institute for Health Research (NIHR) Oxford Biomedical Research Centre (BRC). The views expressed are those of the author(s) and not necessarily those of the NHS, the NIHR or the Department of Health. ACB is an NIHR Senior Investigator and supported by the NIHR Manchester Biomedical Research Centre. LVW holds a GSK/British Lung Foundation Chair in Respiratory Research. The authors are grateful to Dr. Iris Fischer for helpful edits.

Competing interests: The authors declare no competing interests. 


\section{FIGURE LEGENDS}

Figure 1: The translational potential of complex disease genomics.

Improvements to human health (inner circle) are achieved through various enabling milestones at different translational axes (outer circle).

\section{Figure 2: Identifying therapeutic targets.}

(A) Effector transcripts identified at genetic signals are genetically manipulated to recapitulate in vivo effects on gene expression (e.g. CRIPSR knockdown, over-expression) in human cell lines (e.g. IPS cell-derived models) and in animal models, which can be phenotyped. (B) Additional alleles are identified using sequence data and assessed for their relationship to disease risk or related-traits. To provide insight on the therapeutic window, in vitro functional severity and clinical severity are explored to establish the relationship between target perturbation and outcome. Potential adverse on-target effects are investigated using genome-wide datasets for other disorders (PheWAS). (C) Examples of therapeutic targets confirmed or identified by human GWAS.

\section{Figure 3: Pathway to identifying causal variants.}

Trans-ethnic fine-mapping is used to refine genetic association signals and genomic annotation to prioritize likely causal variants. Variants in regulatory elements (e.g. enhancers) are mapped to the promoters and transcripts they regulate through conformation capture approaches (e.g. promoter $\mathrm{Hi}-\mathrm{C}$ ). Expression QTL mapping can provide additional evidence for the effector transcripts and the direction of effect (e.g. increase or decrease in transcript levels).

\section{REFERENCES}

1. J. P. Clancy, M. Jain, Personalized medicine in Cystic Fibrosis. Am. J. Respir. Crit. Care Med. 186, 593-597 (2012).

2. C. H. June, R. S. O'Connor, O. U. Kawalekar, S. Ghassemi, M. C. Milone, CAR T cell immunotherapy for human cancer. Science 359, 1361-1365 (2018).

3. K. Zhou et al., Variation in the glucose transporter gene SLC2A2 is associated with glycemic response to metformin. Nature Genet. 48, 1055-1059 (2016).

4. A. Y. Dawed et al., Variation in the Plasma Membrane Monoamine Transporter (PMAT, Encoded in SLC29A4) and Organic Cation Transporter 1 (OCT1, Encoded in SLC22A1) and Gastrointestinal Intolerance to Metformin in Type 2 Diabetes: An IMI DIRECT Study. Diabetes Care, dc182182 (2019).

5. N. J. Schork, Personalized medicine: Time for one-person trials. Nature 520, 609-611 (2015).

6. National Institutes of Health (NIH), Overview of the Accelerating Medicines Partnership (AMP) Rheumatoid Arthritis and Lupus Network (available at https://www.niams.nih.gov/grants-funding/funded-research/acceleratingmedicines/overview).

7. Medical Research Council (MRC), Precision Medicine (available at https://mrc.ukri.org/research/initiatives/precision-medicine/research/).

8. J. Massey et al., Genome-wide association study of response to tumour necrosis factor inhibitor therapy in rheumatoid arthritis. Pharmacogenomics J. 18, 657-664 (2018).

9. J. Bluett et al., Impact of inadequate adherence on response to subcutaneously administered anti-tumour necrosis factor drugs: results from the Biologics in Rheumatoid Arthritis Genetics and Genomics Study Syndicate cohort. Rheumatology 54, 494-499 (2014).

10. N. Dand et al., HLA-C*06:02 genotype is a predictive biomarker of biologic treatment response in psoriasis. J. Allergy Clin. Immunol., doi:10.1016/j.jaci.2018.1011.1038 (2018).

11. S. Viatte et al., Association of HLA-DRB1 haplotypes with rheumatoid arthritis severity, mortality, and treatment response. JAMA 313, 1645-1656 (2015). 
12. M. A. Seibold et al., A common MUC5B promoter polymorphism and Pulmonary Fibrosis. $N$. Engl. J. Med. 364, 1503-1512 (2011).

13. U. Styrkarsdottir et al., Whole-genome sequencing identifies rare genotypes in COMP and CHADL associated with high risk of hip osteoarthritis. Nature Genet. 49, 801-805 (2017).

14. I. Moltke et al., A common Greenlandic TBC1D4 variant confers muscle insulin resistance and type 2 diabetes. Nature 512, 190-193 (2014).

15. E. A. King, J. W. Davis, J. F. Degner, Are drug targets with genetic support twice as likely to be approved? Revised estimates of the impact of genetic support for drug mechanisms on the probability of drug approval. bioRxiv, 513945 (2019).

16. M. R. Nelson et al., The support of human genetic evidence for approved drug indications. Nature Genet. 47, 856-860 (2015).

17. I. K. Kotowski et al., A spectrum of PCSK9 alleles contributes to plasma levels of low-density lipoprotein cholesterol. Am. J. Hum. Genet 78, 410-422 (2006).

18. S. Pushpakom et al., Drug repurposing: progress, challenges and recommendations. Nat. Rev. Drug Discov. 18, 41-58 (2018).

19. I. Tachmazidou et al., Identification of new therapeutic targets for osteoarthritis through genome-wide analyses of UK Biobank data. Nature Genet. 51, 230-236 (2019).

20. The Encode Project Consortium et al., An integrated encyclopedia of DNA elements in the human genome. Nature 489, 57-74 (2012).

21. Roadmap Epigenomics Consortium et al., Integrative analysis of 111 reference human epigenomes. Nature 518, 317-330 (2015).

22. H. G. Stunnenberg et al., The International Human Epigenome Consortium: A blueprint for scientific collaboration and discovery. Cell 167, 1145-1149 (2016).

23. E. R. Gamazon et al., Using an atlas of gene regulation across 44 human tissues to inform complex disease- and trait-associated variation. Nature Genet. 50, 956-967 (2018).

24. A. Mahajan et al., Fine-mapping type 2 diabetes loci to single-variant resolution using highdensity imputation and islet-specific epigenome maps. Nature Genet. 50, 1505-1513 (2018).

25. O. Rozenblatt-Rosen, M. J. T. Stubbington, A. Regev, S. A. Teichmann, The Human Cell Atlas: from vision to reality. Nature 550, 451-453 (2017).

26. A. V. Khera et al., Genome-wide polygenic scores for common diseases identify individuals with risk equivalent to monogenic mutations. Nature Genet. 50, 1219-1224 (2018).

27. N. Shrine et al., New genetic signals for lung function highlight pathways and chronic obstructive pulmonary disease associations across multiple ancestries. Nature Genet. 51, 481-493 (2019).

28. A. V. Khera et al., Polygenic prediction of weight and obesity trajectories from birth to adulthood. Cell 177, 587-596.e589 (2019).

29. E. Evangelou et al., Genetic analysis of over 1 million people identifies 535 new loci associated with blood pressure traits. Nature Genet. 50, 1412-1425 (2018).

30. L. J. Laslett et al., The worldwide environment of cardiovascular disease: Prevalence, diagnosis, therapy, and policy issues: A report from the American College of Cardiology. J. Am. Coll. Cardiol. 60, S1-S49 (2012).

31. G. Rose, The strategy of preventive medicine. (Oxford University Press, Oxford, 1992), pp. xii $+135$.

32. D. G. Clayton, Prediction and interaction in complex disease genetics: Experience in Type 1 Diabetes. PLOS Genet. 5, e1000540 (2009).

33. B. Feigl, C. P. Morris, The challenge of predicting macular degeneration. Curr. Med. Res. Opin. 27, 1745-1748 (2011).

34. A. Warwick, A. Lotery, Genetics and genetic testing for age-related macular degeneration. Eye 32, 849-857 (2018). 
35. T. J. Heesterbeek et al., Genetic risk score has added value over initial clinical grading stage in predicting disease progression in age-related macular degeneration. Sci. Rep. 9, 6611 (2019).

36. F. Dudbridge, Power and predictive accuracy of polygenic risk scores. PLOS Genet. 9, e1003348 (2013).

37. A. V. Khera, C. A. Emdin, S. Kathiresan, Genetic risk, lifestyle, and coronary artery disease. N. Engl. J. Med. 376, 1192-1195 (2017).

38. L. C. Rutten-Jacobs et al., Genetic risk, incident stroke, and the benefits of adhering to a healthy lifestyle: cohort study of 306473 UK Biobank participants. BMJ 363, k4168 (2018).

39. J. A. Sparks et al., Disclosure of personalized rheumatoid arthritis risk using genetics, biomarkers, and lifestyle factors to motivate health behavior improvements: A randomized controlled trial. Arthrit. Care Res. 70, 823-833 (2018).

40. M. S. Udler, M. I. McCarthy, J. C. Florez, A. Mahajan, Genetic risk scores for diabetes diagnosis and precision medicine. Endocr. Rev., doi:10.1210/er.2019-00088 (2019).

41. A. L. Jorgensen et al., Implementation of genotype-guided dosing of warfarin with point-ofcare genetic testing in three UK clinics: a matched cohort study. BMC Med. 17, 76 (2019).

42. A. R. Martin et al., Clinical use of current polygenic risk scores may exacerbate health disparities. Nature Genet. 51, 584-591 (2019).

43. A. Giri et al., Trans-ethnic association study of blood pressure determinants in over 750,000 individuals. Nature Genet. 51, 51-62 (2019).

44. E. Zeggini, A. L. Gloyn, T. Hansen, Insights into metabolic disease from studying genetics in isolated populations: stories from Greece to Greenland. Diabetologia 59, 938-941 (2016).

45. D. Gurdasani et al., The African Genome Variation Project shapes medical genetics in Africa. Nature 517, 327-332 (2014).

46. E. Wheeler et al., Impact of common genetic determinants of Hemoglobin A1c on type 2 diabetes risk and diagnosis in ancestrally diverse populations: A transethnic genome-wide meta-analysis. PLOS Med. 14, e1002383 (2017).

47. B. S. Glicksberg, K. W. Johnson, J. T. Dudley, The next generation of precision medicine: observational studies, electronic health records, biobanks and continuous monitoring. Hum. Mol. Genet. 27, R56-R62 (2018).

48. F. E. Dewey et al., Genetic and pharmacologic inactivation of ANGPTL3 and cardiovascular disease. N. Engl. J. Med. 377, 211-221 (2017).

49. L. T. Elliott et al., Genome-wide association studies of brain imaging phenotypes in UK Biobank. Nature 562, 210-216 (2018).

50. L. J. Corbin et al., Formalising recall by genotype as an efficient approach to detailed phenotyping and causal inference. Nature Commun. 9, 711 (2018).

51. P. R. Njølstad et al., Roadmap for a precision-medicine initiative in the Nordic region. Nature Genet. 51, 924-930 (2019).

52. A. R. Bentley et al., Multi-ancestry genome-wide gene-smoking interaction study of 387,272 individuals identifies new loci associated with serum lipids. Nature Genet. 51, 636-648 (2019). 


\section{Figure 1}

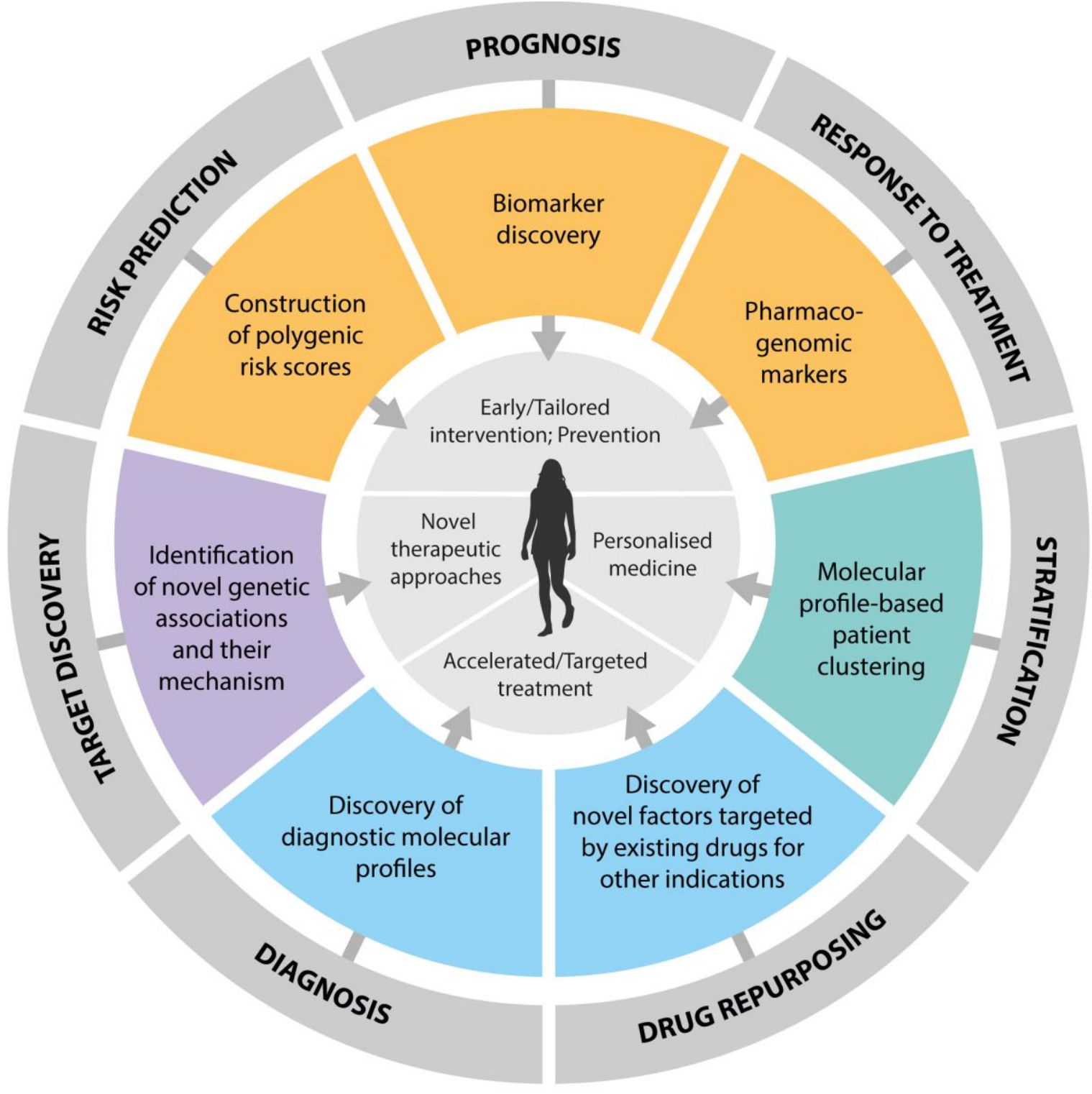


Figure 2

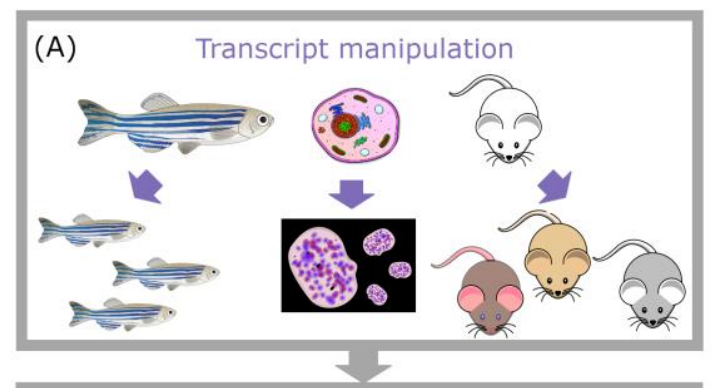

(C)

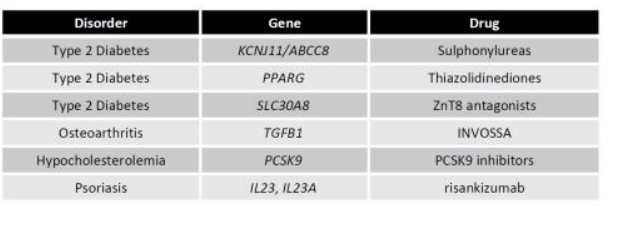

(B)

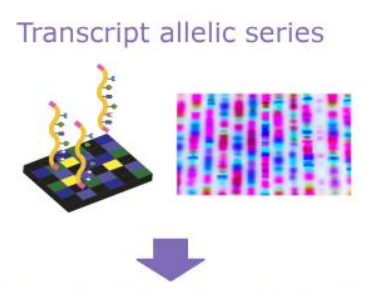

Target efficacy - Phenotype \& Functional Severity

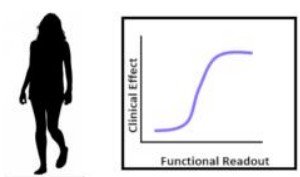

Target Safety - Pleiotropy, PheWAS 
Figure 3

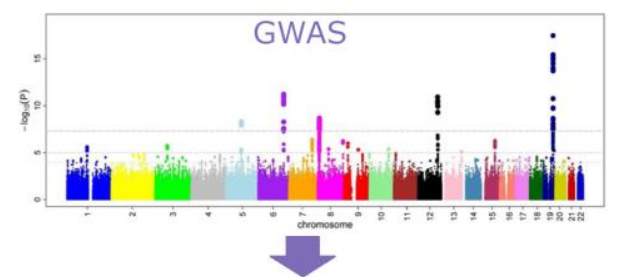

Fine mapping

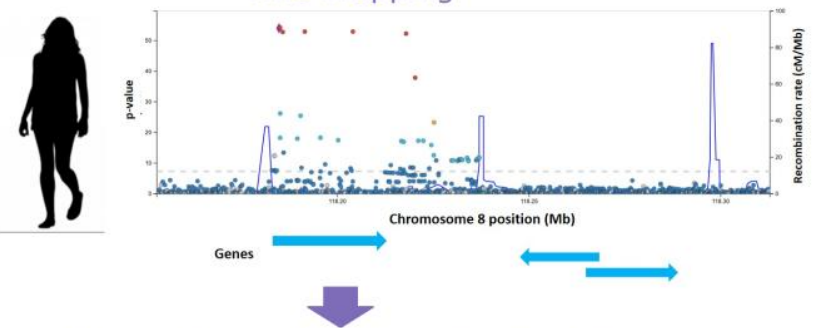

Integration with epigenomic annotation

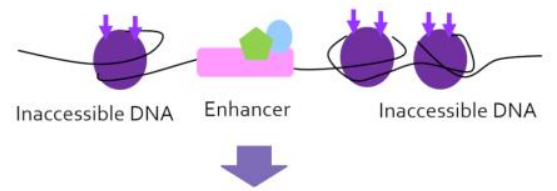

Linking regulatory annotation to transcripts

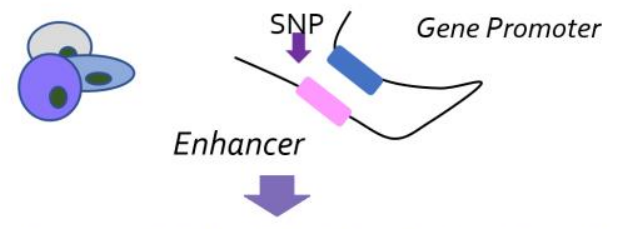

Effector transcript identification \& direction of effect

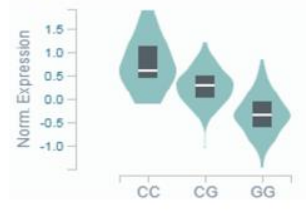

